\title{
Erratum: Role of Tensorial Electronic Friction in Energy Transfer at Metal Surfaces [Phys. Rev. Lett. 116, 217601 (2016)]
}

Mikhail Askerka, Reinhard J. Maurer, Victor S. Batista, and John C. Tully

(Received 10 July 2017; published 8 August 2017)

DOI: 10.1103/PhysRevLett.119.069901

In this Letter, we reported time-dependent perturbation theory (TDPT) calculations of relaxation rates for a hydrogen atom adsorbed on a $\mathrm{Pd}(100)$ surface. These results, as reported, are correct. However, we have found numerical errors in the relaxation rates calculated as a comparison with the local density friction approximation (LDFA) that we have reported in Fig. 1 and Fig. 2 of this Letter. Here, we provide versions of Fig. 1 and Fig. 2 with corrected LDFA values. Other LDFA results in the manuscript are affected. These corrected results agree better in absolute value with relaxation rates calculated from TDPT, although the overestimation of electronic friction with respect to TDPT persists due to differences in absolute

\begin{tabular}{|c|c|c|c|}
\hline & Top View & Side View & \multicolumn{2}{|c|}{ Friction $\tilde{\Lambda}\left(\mathrm{ps}^{-1}\right)$} \\
\hline & & \\
\hline
\end{tabular}

FIG. 1. (left) Hydrogen atom on $\operatorname{Pd}(100)$ as viewed from $x y$ (top view) and $y z$ (side view) planes for the hollow, bridge, top, and subsurface sites. Dimmed circles point at the positions of the hydrogen atom when it is not directly visible at current view. The depicted arrows are proportional to the magnitude of electronic friction. (right) Components of the mass-weighted friction tensor (in $\mathrm{ps}^{-1}$ ) along Cartesian directions and the isotropic rate as given by LDFA $\tilde{\Lambda}_{\mathrm{LDFA}}$. 


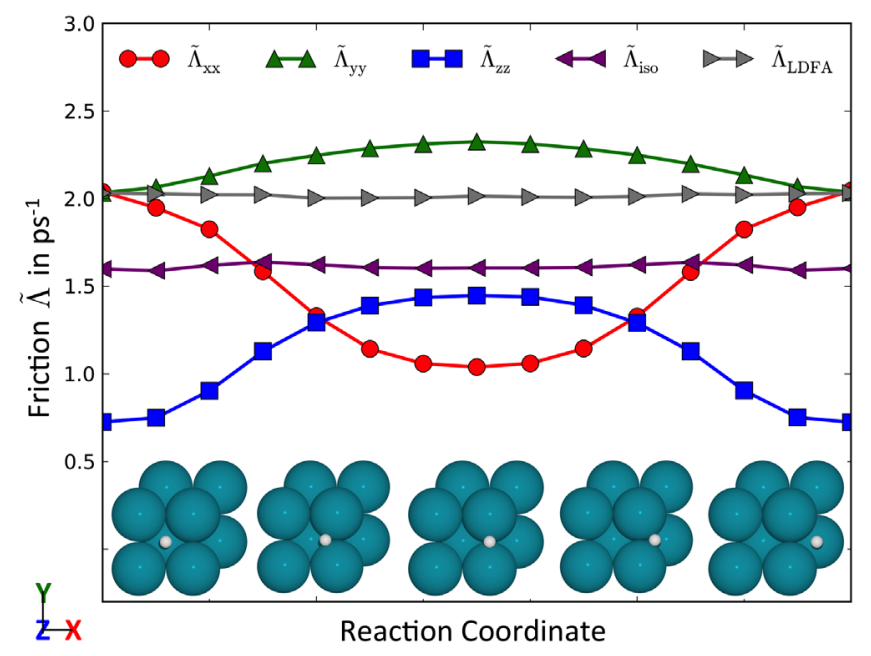

FIG. 2. Cartesian components of the mass-weighted friction tensor $\tilde{\boldsymbol{\Lambda}}$ (in ps ${ }^{-1}$ ) of a hydrogen atom on $\mathrm{Pd}(100)$ as it moves from one equilibrium hollow site to another (first and last points) across a bridge site (middle point). Shown are the relaxation rates along the three Cartesian components, the rate as given by the average of trace of the mass-weighted friction tensor $\left(\tilde{\Lambda}_{\text {iso }}=\operatorname{Tr}(\tilde{\Lambda}) / 3\right)$, and the isotropic rate as given by LDFA $\tilde{\Lambda}_{\mathrm{LDFA}}$.

relaxation rates and the lack of directional anisotropy and friction-induced mode coupling in LDFA. Our conclusions are, therefore, not affected.

The authors are grateful to A. Kandratsenka (Max Planck Institute for Biophysical Chemistry, Göttingen) for pointing this error out. 